\title{
Performance of UWB Modulation Schemes and UWB Pulses for Outdoor Power Line Communications
}

\author{
Ana Luiza S. Ferreira, Renato Machado, and Moisés V. Ribeiro
}

\begin{abstract}
Resumo-A viabilidade do uso de pulsos de banda ultra-larga (UWB - ultrawideband) e diferentes esquemas de modulação UWB para a transmissão de dados através das redes de distribuição de energia elétrica, mais conhecidas por canais PLC (power line communications), são discutidas nesta contribuição. Resultados de simulação para canais outdoor PLC na baixa tensão, invariantes no tempo, corrompidos pela presença de ruído impulsivo, revelam que o desempenho de um sistema UWB-PLC depende fortemente de um bom projeto do pulso UWB, que por sua vez deve estar atrelado ao perfil de atenuação da resposta em freqüência do canal PLC. Além disso, tais resultados indicam que o esquema PPM (pulse position modulation) oferece o melhor desempenho em termos de BER (bit error rate) e a menor taxa de transmissão. Outras observações apresentadas no presente trabalho evidenciam a necessidade de uma análise mais detalhada sobre a comunicação UWB para sistemas PLC.
\end{abstract}

Palavras-Chave-Comunicação por pulso de banda ultralarga, comunicação via rede elétrica, pulso de banda ultra-larga, ruído impulsivo.

Abstract-The suitability of the well-known ultra wideband (UWB) pulses and different UWB modulation schemes for power line communications (PLC) are addressed in this contribution. Numerical results for low-voltage outdoor time-invariant PLC channels corrupted by the presence of impulsive noises reveal that UWB-PLC system performance is related to a good tradeoff between UWB pulse design and PLC channel attenuation profile. As a result, it is difficult to state that only one UWB pulse is appropriate for a general UWB-PLC system. In addition, the numerical results indicate that the PPM (pulse position modulation) offers the best performance in terms of BER but the lowest bit-rate. Other analysis provided in this contribution reveals the need for a deep investigation of UWB communication for PLC systems.

Keywords - Impulsive noise, power line communications, UWB communications, UWB pulse.

\section{INTRODUCTION}

In recent years, pulse-based ultrawideband (UWB) communications has been deeply investigated to offer new and advantageous tradeoff in terms of performance, reliability, and coverage for high-speed last meters communication systems [1]. UWB communications is mainly characterized by transmitting short pulses in time and highly spread in frequency. Therefore, UWB pulses have very low power spectral density. In spite of the attained development in this field not only for wireless but also to wireline fields, many issues have to be addressed for advances in UWB technology [2]. Among them,

A. L. S. Ferreira, R. Machado, and M. V. Ribeiro, are with the Department of Electrical Engineering, Federal University of Juiz de Fora, MG, Brazil, E-mails: analuiza@ieee.org, \{renatomachado,mribeiro\}@engenharia.ufjf.br. the suitability of UWB pulses is a key issue to be investigated because of the variability of communication channel and noise.

Regarding an emerging wireline field widely named power line communication (PLC) [3], few attention has been put on UWB for devising next generation of PLC technology [4][7]. The reason for that resides on the fact that UWB for PLC systems is in the infancy stage and commercial application are not well defined yet because of the cost, suitability, and performance comparison among UWB, multicarrier, singlecarrier based PLC systems. However, the investigation of UWB for PLC system is a very interesting and timely issue because it can offer direction for the most appropriate use of UWB communication in PLC field.

In [4]-[7] different aspects of UWB communication were investigated. However, the performance comparison of UWBPLC system based on the choice of UWB pulse shapes as well as modulation approaches is an open question in the literature. It is important to mention that such analysis is not a simple one because PLC channels can vary considerably among outdoor (high-voltage (HV), medium-voltage (MV), low-voltage (LV) ) and indoor (residential, commercial, industrial, and vehicular) electric networks. Then, a priori, it is not possible to state that the performance comparison for specific electric networks cannot be generalized at all.

As UWB pulse design is a key and timely issue to advance UWB-PLC systems, performance comparison of these systems based on the choices of pulse shapes and modulation scheme, such as pulse shape modulation (PSM), pulse position modulation (PPM) and and pulse amplitude modulation (PAM) are presented and discussed in this contribution. UWB pulses considered are Gaussian pulses (GP) and modified Hermite pulses (MHP) [8]. The target PLC channels are outdoor and LV electric networks, which comprise the power lines among residential power meters and distribution transformer. Based on linear and time-invariant (LTI) PLC channels and additive impulsive Gaussian noise (AIGN) models, numerical results in terms of bit error rate (BER) are provided and analyzed. For this kind of PLC channels, the numerical results reveal that if the design of pulses for UWB-PLC systems take into account partial channel information, then improved performance can be achieved. Finally, but not the last, the numerical results indicate that PPM scheme is the one offering the best performance in terms of BER.

This paper is organized as follows. Section II describes the UWB PLC system considered in the simulations. Section II-A focus on the description of some UWB pulse shapes. Section 
III evaluates and compares the simulation performances of different kinds of UWB pulses in the presence of the PLC channel and impulsive noise. Finally, Section IV presents some concluding remarks and final comments.

\section{UWB PLC: SYSTEM MODEL}

A system model for $M$-ary UWB-PLC is depicted in Fig. 1. For this system model, the $j$ th message $m_{j}$ composed of $k=\log _{2} M$ bits is transmitted in each UWB symbol. It is assumed that UWB pulse shapes can be GP or MHP and modulation schemes are PAM, PPM, and PSM [2]. Based on these modulation schemes and the pulse shapes, a general formulation of UWB pulses considered in this contribution can be expressed by

$$
x_{\psi}(t)=\sum_{j=-\infty}^{\infty} \alpha_{a, i, j} w_{i}^{s}\left(t-j T_{f}-\delta_{p, j}\right), i=1,2, \ldots, M,
$$

where $w_{i}^{s}($.$) denotes s$-order GP or MHP, $T_{f}$ the frame period, $\alpha_{a, i, j}$ and $\delta_{p, j}$ the amplitude and the delay of the $j$ th symbol that contains the $i$ th pulse, respectively. As a result, PAM, PPM, and PSM modulations can be derived from the general formulation based on the values in Tab. I. In Tab. I, $N$ denotes the constellation size. Different values on Tab. I can offer other UWB modulations (PAM-PPM, PAM-PSM, etc) [9]. The parameter $\alpha_{a, i, j}$ is a constant value for PPM and PSM modulations and it varies for PAM modulation. $\delta_{p, j}$ may assume different values for PPM modulation while it is a constant for other modulations.

TABLE I

NECESSARY CONDITIONS FOR DERIVING PAM, PPM, AND PSM MODULATIONS.

\begin{tabular}{|c||c|c|c|}
\hline Modulation & PAM & PPM & PSM \\
\hline \hline $\mathrm{a}$ & $1,2, \ldots \mathrm{N}$ & 1 & 1 \\
\hline $\mathrm{p}$ & 1 & $j=1, \ldots, \mathrm{N}$ & 1 \\
\hline $\mathrm{s}$ & 1 & 1 & $j=1, \ldots, \mathrm{N}$ \\
\hline
\end{tabular}

The frame period of each transmitted UWB symbol is $T_{f} \geq$ $T_{p}+T_{g}$, where $T_{p}$ and $T_{g}$ denote time duration of UWB pulse and guard period, respectively. Usually, it is assumed $T_{g} \geq T_{h}$, where $T_{h}$ denotes the time period of PLC channel impulse response. Due to the adopted frame period for each UWB pulse transmitted, equalization technique is not used at the receiver side, once the received signal at the receiver side does not present intersymbol interference.

The receiver's baseband input is expressed by

$$
y(t)=\tilde{y}(t)+v(t)=x_{\psi}(t) \star h(t)+v(t),
$$

where $\widetilde{y}(t)$ denotes the PLC channel output free of noise, $x_{\psi}(t)$ the transmitted UWB pulses, $\star$ convolution operator, $h(t)$ the LTI PLC channel model, and $v(t)$ the AIGN model at the PLC channel output.

The frequency response of the top-down PLC channel model considered in the simulations is expressed by [10]

$$
H(f)=\sum_{i=1}^{N} g_{i} \cdot e^{-\left(a_{0}+a_{i} f^{k}\right) d_{i}} \cdot e^{-j 2 \pi f\left(d_{i} / v_{p}\right)},
$$

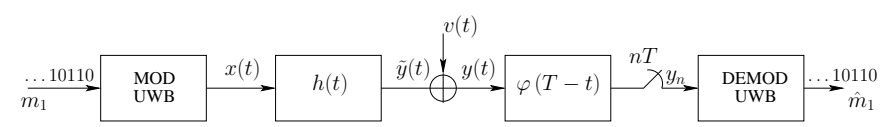

Fig. 1. Block diagram of the UWB system model.

where $g_{i}$ represents a weighting factor, $e^{-\left(a_{0}+a_{i} f^{k}\right) d_{i}}$ is the attenuation portion and $e^{-j 2 \pi f\left(d_{i} / v_{p}\right)}$ is the delay portion. The parameters of each portion are: $N$ (number of propagations paths); $i$ (number of path, where the path with the shortest delay has the index $i=1$ ); $a_{0}$ e $a_{1}$ (attenuation parameters); $k$ (exponent of the attenuation factor (typical values are between 0.5 and 1$)$ ); $g_{i}$ (weighting factor for path $i$, in general complex, can be considered as combination of the involved reflection and transmission factors); $d_{i}$ (length of path $i$ ); $v_{p}$ (the speed of light divided by the mean square of the dielectric constant).

The energy normalized (norm-2) frequency response attenuation profiles of two outdoor PLC channels are portrayed in Fig. 2 [10]. Their bandwidths range from 0 up to $50 \mathrm{MHz}$. Channels \#1 and \#2 are associated with typical users located near and far from the MV-LV transformers, respectively.

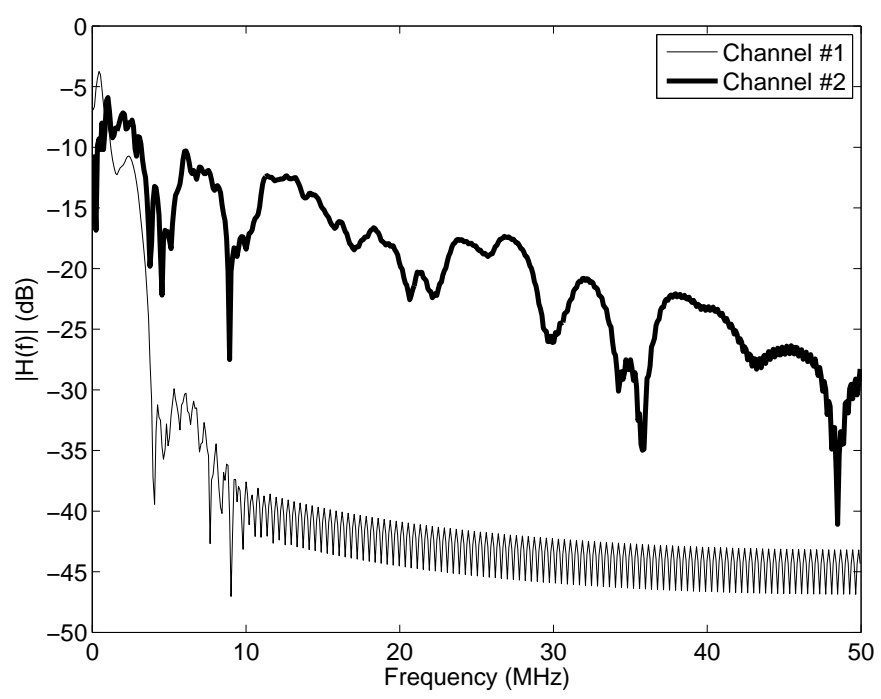

Fig. 2. Normalized frequency response of PLC channels.

The AIGN model can be given by [4]

$$
v(t)=v_{b k g r}(t)+v_{i m p}(t),
$$

where $v_{b k g r}(t)$ and $v_{i m p}(t)$ denote the background and impulsive noise components, respectively. These components are generated according to the two-term Gaussian model whose probability density function is defined as $p_{\eta}=(1-$ $\varepsilon) \mathcal{N}\left(0, \sigma_{1}{ }^{2}\right)+\varepsilon \mathcal{N}\left(0, \sigma_{2}{ }^{2}\right)$ [4]. The first term gives the $v_{b k g r}(t)$ with variance $\sigma_{1}{ }^{2}$ and the second term gives the $v_{i m p}(t)$ with variance $\sigma_{2}^{2}=100 \sigma_{1}^{2}$ [4]. The occurrence probability is $\varepsilon=0.01$. It has been assumed that each Gaussian process lasts for a period of time corresponding to four frames. Fig. 3 illustrates a realization of additive noise when $\sigma_{1}{ }^{2}=-3 \mathrm{~dB}$.

For the receiver, we consider time-shifting-coherent communication. For decoding, we use the $M$-ary correlation receiver with perfect synchronization for each modulation scheme. 


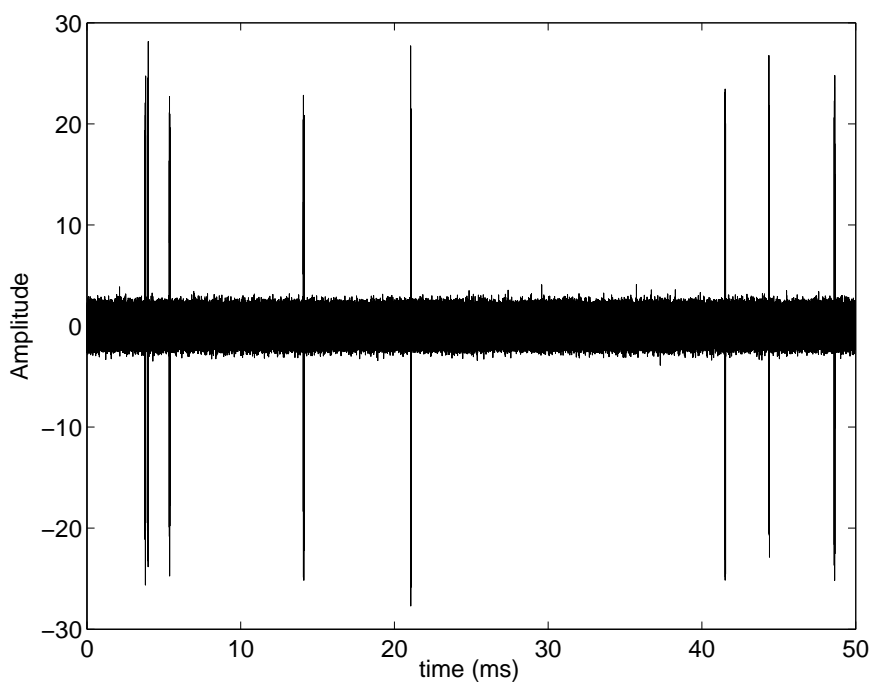

Fig. 3. AIGN realization.

Although other symbol detections are on the literature [11], in this contribution the detection is based on correlation. In this case, we need to correlate the received signals with $M, a$ priori, known UWB pulses. The output of the $j$ th correlation is given by

$$
\begin{array}{r}
d\left(j, T_{s}, i\right)=\int_{j T_{f}+T_{s}}^{j T_{f}+T_{s}+T_{p}} y(t) \alpha_{a, i, j} w_{i}^{s}\left(t-j T_{f}-\delta_{p, j}\right) d t \\
=\int_{j T_{f}+T_{s}}^{j T_{f}+T_{s}+T_{p}}\left[x_{\psi}(t) \star h(t)\right] \alpha_{a, i, j} w_{i}^{s}\left(t-j T_{f}-\delta_{p, j}\right) d t+ \\
\int_{j T_{f}+T_{s}}^{j T_{s}+T_{p}} v(t) \alpha_{a, i, j} w_{i}^{s}\left(t-j T_{f}-\delta_{p, j}\right) d t, i=1,2, \ldots, M,
\end{array}
$$

where $T_{s}$ denotes the synchronization instant. Then, the estimation of the $j$ th transmitted message is

$$
\hat{m}_{j}=\max _{i=1,2, \ldots, M} d\left(j, T_{s}, i\right) .
$$

\section{A. UWB Pulses}

Pulse shapes that are commonly used in UWB theoretical studies include Gaussian, Laplacian, Rayleigh, or modified Hermitean pulses [2]. In this work, we have focused on GP and MHP.

The gaussian waveforms have mathematical definition similar to the Gauss function [8]. The basis of these waveforms is a Gaussian pulse represented by

$$
w_{G P_{0}}=K_{0} e^{-(t / \tau)^{2}},
$$

where $K_{0}$ is a constant which controls the energy of the pulse and $\tau$ is a time-scale factor. More waveforms can be created by a sort of high-pass filtering of this Gaussian pulse. Filtering acts in a manner similar to taking the derivative of (7). Then, GP of $n$th order are given by

$$
w_{G P_{n}}=K_{n} \frac{d^{n}}{d t} e^{-(t / \tau)^{2}},
$$

where $n=0,1,2, \ldots,-\infty<t<\infty$ and $K_{n}$ are constants which vary with the energy $E_{n}$ of each pulse. The constant $\tau$ is a time-scale factor.

MHP [8] are inferred from the Hermite polynomials. These polynomials have been modified to became orthogonal. The general formula of the MHP of $n$th order is expressed by

$$
w_{M H P_{n}}=K_{n} e^{-t^{2} / 4 \tau^{2}} n ! \sum_{i=0}^{n / 2}\left(-\frac{1}{2}\right)^{i} \frac{(t / \tau)^{n-2 i}}{(n-2 i) ! i !},
$$

where $n=0,1,2, \ldots,-\infty<t<\infty$ and $K_{n}$ are constants which vary with the energy $E_{n}$ of each pulse. The constant $\tau$ is a time-scale vector. The constants $K_{n}$ vary with the energy of the pulse.

Fig. 4 shows time domain waveforms of MHP designed to occupy bandwidth ranging from 0 up to $12.5 \mathrm{MHz}$ and fom 0 up to $50 \mathrm{MHz}$. In this figure, it is considered that the energy of both MHP pulses are equal to 1 .

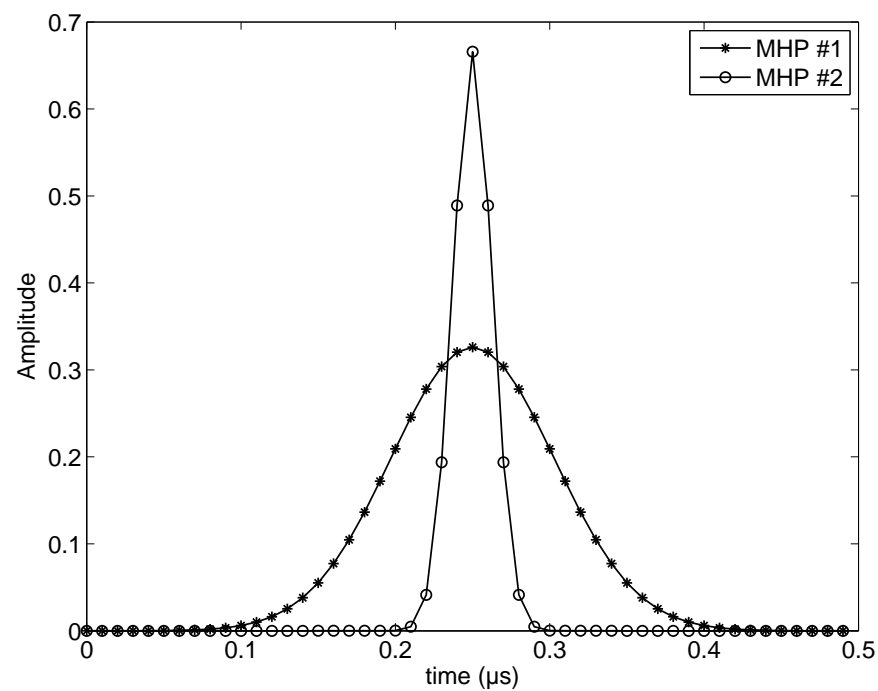

Fig. 4. $-*-$ and $-0-$ lines denote MHP of order zero designed to occupy $12.5 \mathrm{MHz}$ and $50 \mathrm{MHz}$ of bandwidth, respectively.

\section{Simulation Results}

To carry out computational simulation with the described UWB-PLC system, GP and MHP have been designed to occupy bandwidth ranging from 0 up to $12.5 \mathrm{MHz}, 25 \mathrm{MHz}$, or $50 \mathrm{MHz}$. The amplitude spectrum of the designed pulses decays to $-50 \mathrm{~dB}$ at $12.5 \mathrm{MHz}, 25 \mathrm{MHz}$, or $50 \mathrm{MHz}$. The GP and MHP orders are equal to $0,1,2$, and 3 . The designed pulses obey the Federal Communications Commission (FCC) rules which states that any signal or pulse satisfying a minimum of 0.20 fractional bandwidth can be considered as an UWB signal [1]. In all simulations, the pulses are transmitted in baseband and it is considered that the synchronization is ideal at the receiver side. What is more, the parameter energy per bit to noise power spectral density ratio $\left(E_{b} / N_{0}\right)$, showed in all the simulation results below, represents the ratio between the energy of the pulse and the power spectral density of the background noise $\left(v_{b k g r}(t)\right)$. The energy of the transmitted 
UWB symbols are normalized to 1 . The channel impulse has energy normalized equal to 1 . Also, the bit-rate of the simulation results showed in Fig. 5, 6 and 7 have been fixed to the same value (1.54 Mbps).

To verify how the UWB-PLC system performance can be subjected to spectral correlation between frequency responses of PLC channel and UWB pulses, several simulations were carried out. In this case, GP and MHP have been designed to occupy bandwidth ranging from 0 up to $12.5 \mathrm{MHz}, 25 \mathrm{MHz}$, or $50 \mathrm{MHz}$. The amplitude spectrum of the designed pulses decays to $-50 \mathrm{~dB}$ at $12.5 \mathrm{MHz}, 25 \mathrm{MHz}$, or $50 \mathrm{MHz}$, and the GP and MHP pulse orders are equal to $0,1,2$, and 3 .

Fig. 5 highlights the performance of UWB-PLC system with those UWB pulse orders that offered the highest performance in terms of BER versus $E_{b} / N_{0}$ when PPM scheme, PLC channel \#2 (see Fig. 2), and AIGN model are considered. For the $12.5 \mathrm{MHz}$ bandwidth, 1st-order pulses offer better performance, while to the other UWB bandwidths, pulses of zero-order provided better performance. It is clearly noticeable in Fig. 2 that as close as frequency response of UWB pulses is concentrated around frequency 0 , the more efficient are the UWB-PLC system performance in terms of BER. In other words, once the outdoor PLC channel is characterized by strong attenuations as the frequency increases (see Fig. 2), the pulses with their spectrums concentrated in low frequencies are expected to be advantageous in comparison with the others due to the reduction of energy losses yield by the PLC channel that act, in this case, as a low-pass filter. That is a very important issue because in outdoor LV networks the distance between the transmitter and receiver is considerable and, as a consequence, the attenuation of the transmitted signal through the PLC channel increases considerably as the frequency increases.

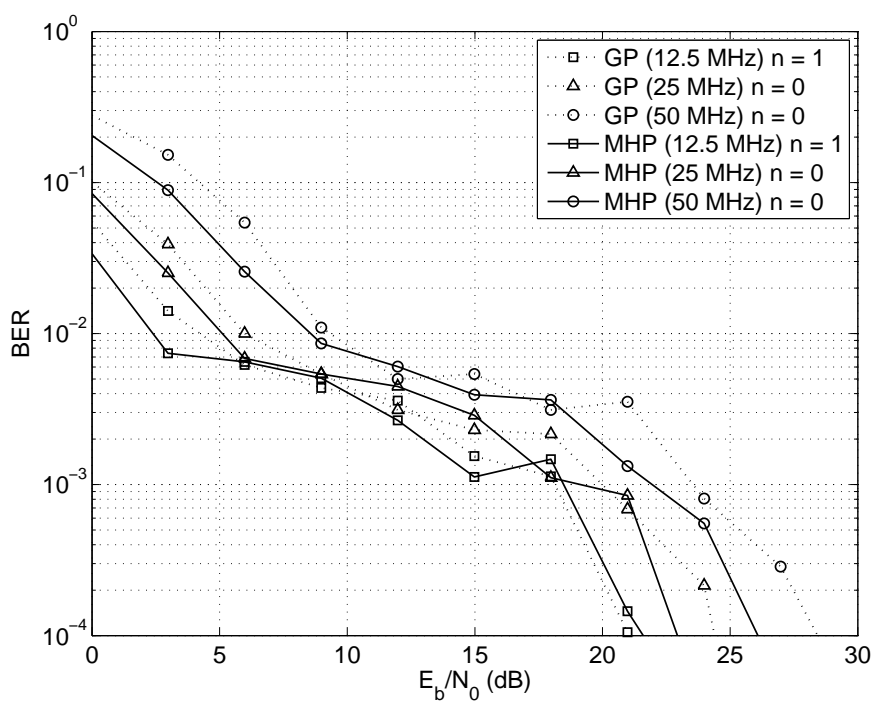

Fig. 5. Performance comparison of UWB-PLC system. UWB pulses are designed with three different bandwidths. PLC channel \#2 and AIGN models are considered.

To compare the behavior of UWB modulation schemes, Fig. 6 shows the performance of PPM, PAM and PSM when GP are transmitted through PLC channel \#2. In this case, GP are designed with $50 \mathrm{MHz}$ bandwidth. Based on the performance results reported in this figure, it is clear that PPM shows better BER performance than PAM for all pulse orders considered in the simulations. Also, PPM with zero- and 1st-orders GP surpasses the performance attained with PSM. These results indicate that PPM is the most robust and appropriate modulation scheme for UWB-PLC system operating in outdoor LTI PLC channels with frequency selectivity and AIGN. Also, they reveal that PAM without channel equalization should be avoided because of its very poor performance. Although the attained results with MHP pulses are not illustrated here, we can state that they present similar results to those depicted in Fig. 6.

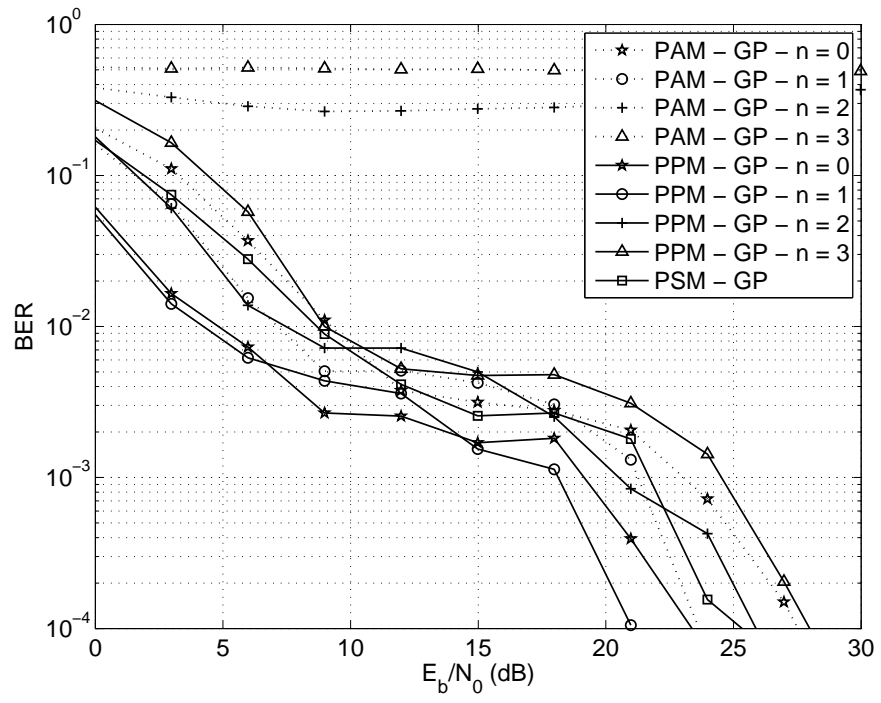

Fig. 6. Performance results of UWB-PLC system designed with MHP and different modulation schemes. PLC channel \#2 and AIGN models are considered.

To analyze which pulse, GP or MHP, is more advantageous to data transmission through the outdoor LTI PLC channel \#2 corrupted by AIGN, Fig. 7 shows performance comparison results when both pulses were designed with 12.5 $\mathrm{MHz}$ bandwidth and the modulation scheme is the PPM. As mentioned before, the results show a close match between attained performance with zero- and 1st-orders GP and zeroand 1st-orders MHP pulses, respectively. On the other hand, the same cannot be noted if one comparatively analyzes 2 ndand 3rd-orders GP and MHP. Finally, the 1st-order MHP and GP achieve the best BER versus $\mathrm{E}_{b} / N_{0}$ performance in comparison to the others.

To verify how the overall performance of UWB-PLC system is impacted by the choice of bandwidth of UWB pulses when the PLC channel switches between two impulse responses, as is presented in [12], some simulation were carried out. In this case, the UWB-PLC system was designed with MHP occupying bandwidths equal to $12.5 \mathrm{MHz}$ and $50 \mathrm{MHz}$, the PLC channel switches between channel \#1 and channel \#2 (see Fig. 2) with probabilities $p_{\# 1}=p_{\# 2}=0.5$ for each UWB symbol period, and the noise is AIGN. In this situation the channel varies as well as the pulse bandwidth, then the bit- 


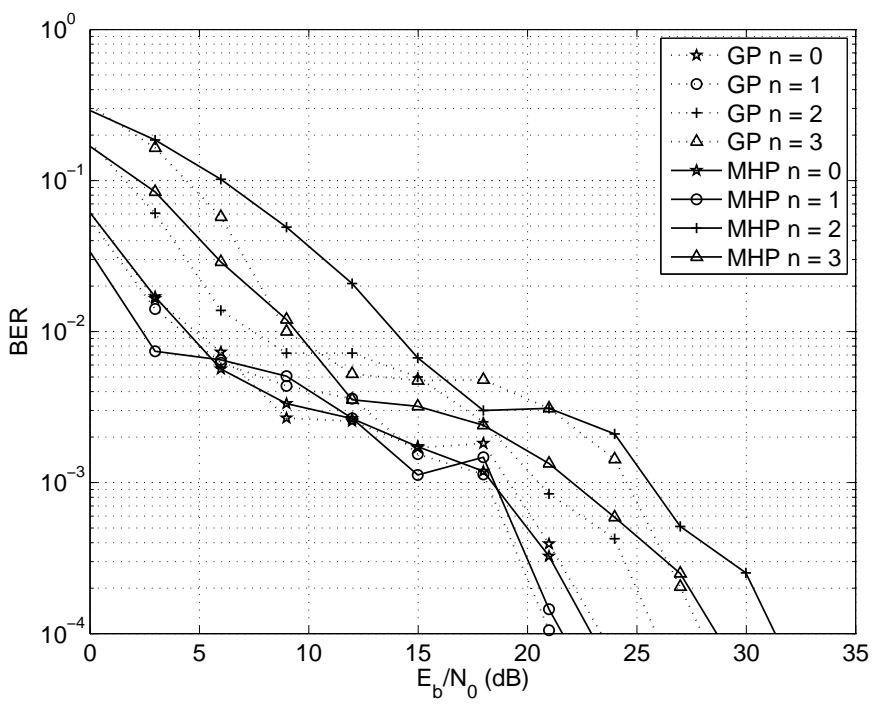

Fig. 7. Performance comparison of UWB-PLC systems designed with GP and MHP pulses. PLC channel \#2 and AIGN models are considered.

rate is not the same as the bit-rate of the previous simulations. This occurs because the PPM pulse has been optimized to show its better performance in each case, what means that the bit-rate modifies according to the pulse bandwidth. Based upon this assumption, three typical scenarios emerge to be analyzed: $i$ ) Case \#1: the UWB-PLC system is designed with MHP occupying $12.5 \mathrm{MHz}$ and offering a bit-rate equal to $0.71 \mathrm{Mbps}$; ii) Case \#2: the same system is now designed with MHP occupying $50 \mathrm{MHz}$ of bandwidth. For case \#2, the attained bit-rate is equal to $1.54 \mathrm{Mbps}$; iii) Case \#3: the UWB-PLC system is designed with two MHP, each of them occupies the bandwidth starting in 0 up to $12.5 \mathrm{MHz}$ or up to $50 \mathrm{MHz}$, respectively. For case \#3, the transmitter, based on a partial channel information, is capable of transmitting the $12.5 \mathrm{MHz}$ or $50 \mathrm{MHz}$ UWB pulse if the transmission channel is \#1 or \#2, respectively. The bit-rate for case \#3 is $0.98 \mathrm{Mbps}$. The performance results, in terms of BER, are presented in Fig. 8. These results indicate that the a good strategy when the channel varies is to design the UWB pulse occupying a fraction of the channel bandwidth because a reduced attenuation of the transmitted pulse is observed at the receiver side. Overall, for case \#1, the best performance in terms of BER versus $E b / N_{0}$ is attained, but the worst in terms of bit-rate. For case \#2, UWB-PLC system offer the worst performance in terms of BER versus $E b / N_{0}$ and the best bit-rate. Intermediate results are achieved for the case \#3. These results indicate that the UWB pulse should be designed based on the minimum energy loss of the transmitted UWB symbol through the PLC channel. This improve the SNR at the receiver side and is the rationale for the results showed in Fig. 8.

\section{CONCLUSION}

The performance comparison of UWB-PLC system for data transmission over outdoor and LV electric networks can offer very interesting directions for further investigation and design

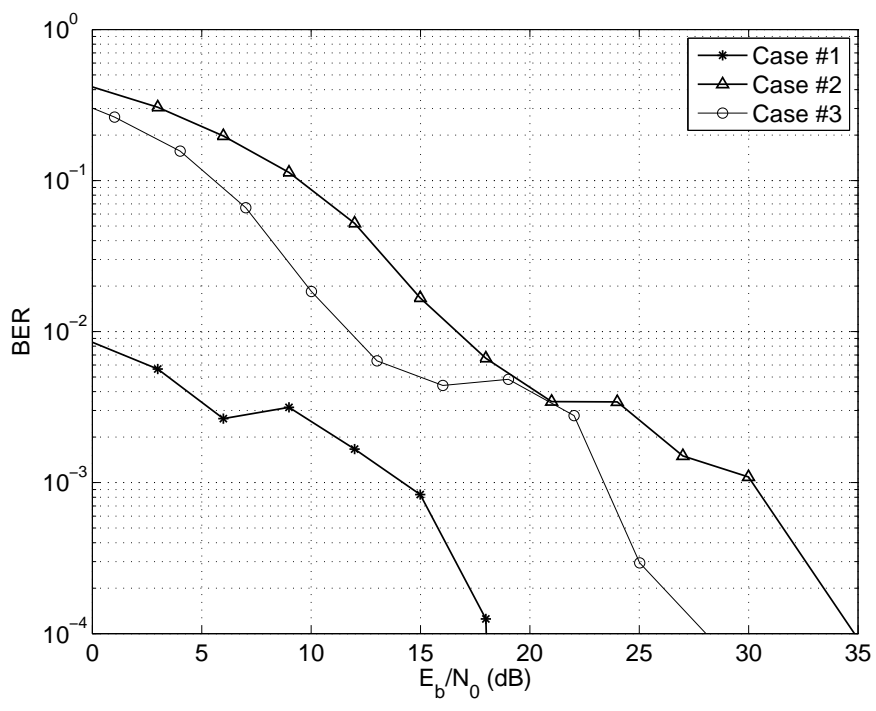

Fig. 8. Performance comparison of UWB-PLC system in cases \#1, \#2 and \#3.

of UWB communication system for PLC applications. Based upon the discussed performance of UWB-PLC system, it could be verified through computational simulations, that severe attenuations yielded by PLC channel in the transmitted UWB pulse when the frequency increases (this is usually occurs in outdoor LV networks), result in poor performance if the UWB pulse occupies all the PLC channel bandwidth. Then, improvements can be attained if the UWB pulses are designed to occupy a fraction of the channel bandwidth that reduces the UWB pulse losses of energy at the receiver side. It means that partial information of PLC channel such as attenuation profile can be very useful to improve UWB-PLC system performance. In addition, PPM scheme can offer the best performance in terms of BER and, consequently, can be a good scheme to be deeply investigated for UWB-PLC systems. Also, it could be verified that PAM is the modulation scheme that should be avoided if channel equalization is not taken into account. Finally, zero- and 1st-order pulses are those ones that can provide the lowest BER versus $E_{b} / N_{0}$ performance in all analyzed scenarios due to the attenuation profile of the frequency response of outdoor PLC channels.

\section{ACKNOWLEDGMENT}

This contribution was supported in part by FAPEMIG, CNPq, CAPES, FINEP, and INERGE.

\section{REFERENCES}

[1] L. Yang and G. B. Giannakis, "Ultra-wideband communications: An idea whose time has come," IEEE Signal Processing Magazine, vol. 21, no. 6, pp. 26-54, November 2004.

[2] M.G. Di Benedetto, T. Kaiser, A. F. Molisch, I. Opermmann, C. Politano, and D. Porcino, UWB Communication System - A Comprehensive Overview, Hindawi Publishing Corporation, New York, 2006.

[3] M. V. Ribeiro, Power line communications: A promising communication system's paradigm for last miles and last meters applications, Book chapter in Telecommunications: Advances and Trends in Transmission, Networking and Applications, Org. Charles C. Cavalcante, Ricardo F. Colares e Paulo Cesar Barbosa, Fundação Edson Queiroz , pp. 133-156, 2006. 
[4] A. M. Tonello, "Wideband impulse modulation and receiver algorithms for multiuser power line communications," EURASIP Journal on Advances in Signal Processing, vol. 2007, no. 17, pp. 1-14, March 2007.

[5] G. Mekuria and H. Hirsch, "UWB pulse transmission over powerline channel," IEEE International Symposium on Power Line Communications and Its Applications (ISPLC), March 2007, pp. 308-313.

[6] G. Mathisen and A. M. Tonello, "WireNet: An experimental system for in-house power line communication," IEEE International Symposium on Power Line Communications and Its Applications (ISPLC), 2006 pp.137-142.

[7] S. Chen, C. C. Chiau, X. Chen, and C. G. Parini, "Characteristic of UWB pulse over powerline communication," Loughborough Antennas and Propagation Conference, 2007, pp. 269-272.

[8] M. Ghavami, L. B. Michael, and R. Kohno, Ultra Wideband Signals and Systems in Communication Engineering, John Wiley \& Sons, England, 2004.
[9] H. Zhang, W. Li and T. A. Gulliver, "Pulse position amplitude modulation for time-hopping multiple-access UWB communications," IEEE Transactions on Communications, vol. 53, no. 8, pp. 1269-1273, August 2005.

[10] M. Zimmermann and K. Dostert, "A multipath model for the powerline channel," IEEE Transactions on Communications, vol. 50, no. 4, pp. 553-559, April 2002

[11] J. A. Lopez-Salcedo and G. Vazquez, "Detection of PPM-UWB random signals," IEEE Transactions on Signal Processing, vol. 56, no. 5, pp. 2003-2016, May 2008.

[12] F. J. Canete, J. A. Cortes, L. Diez, and J. T. Entrambasaguas, "Analysis of the cyclic short-term variation of indoor power line channels," IEEE Journal on Selected Areas in Communications, vol. 24, no. 7, pp. 13271338, July 2006. 